For Debate...

\title{
Pulmonary embolism in surgical patients: 1959-79
}

\author{
C V RUCKLEY, C THURSTON
}

\begin{abstract}
The Edinburgh surgical statistics (audit) have been analysed for the years 1959, 1964, 1969, 1974, and 1979 to determine the trends in pulmonary embolism in surgical patients who died. There was a total of 61038 operations, 1528 postoperative deaths, 804 necropsies, and 158 reported pulmonary emboli. The incidence of embolism diagnosed clinically and at necropsy fell throughout the period. This fall held good after corrections for necropsy rates, prognosis, and proportions of major operations. Although the overall necropsy rate fell from $58 \%$ to $40 \%$, in patients expected to have a good prognosis the rate rose from $68 \%$ to $75 \%$. Necropsy-proved embolism in "good prognosis" patients fell from $0.5 \%$ to $0.15 \%$ per 100 major operations. The main reduction has taken place since most surgeons in the area adopted methods of prophylaxis against venous thrombosis, but a direct relationship is not proved by this study.
\end{abstract}

\section{Introduction}

Pulmonary embolism has been described as the commonest acute lung disorder in hospital patients. ${ }^{1}$ The Registrar General's figures for England and Wales ${ }^{2}$ and for Scotland ${ }^{3}$ have shown substantial increases in incidence over the past 50 years. Much of the research effort and expenditure devoted to this subject in recent years has been prompted by awareness of these trends. To our knowledge there have been no published data showing any contrary evidence. It is still conventional teaching that the incidence is rising. ${ }^{4}$ Is this in fact correct so far as surgical patients are concerned?

This study was undertaken to investigate the incidence of major pulmonary embolism reported in patients dying in surgical units over a 20 -year period.

\section{Methods}

The surgical units of Edinburgh have collected statistics on operations and mortality since Sir James Learmonth began the Saturday morning surgical meetings over 30 years ago. In modern parlance this is a system of clinical audit. Each unit prepares its annual report for presentation at a regular Saturday morning meeting. The report comprises a complete list of the year's operations and of all deaths. The statistics on each death are set out in a relatively standard

University Department of Clinical Surgery, Royal Infirmary, Edinburgh EH3 9YW

C V RUCKLEY, MB, FRCSED, consultant surgeon and part-time senior lecturer, University of Edinburgh

C THURSTON, MB, CHB, surgical registrar (present address: Bangour General Hospital, Broxburn, West Lothian EH52 6LR) format that includes information on the patient's age, sex, time in hospital, time after operation, clinical diagnosis, nature of operation, clinical cause of death, and findings at necropsy. For the purposes of this study the reports of 10 units have been examined for the years $1959,1964,1969,1974$, and 1979, giving a span of 20 years.

The data were derived from the work of seven general surgical units and three specialised units (two urological, one gastrointestinal). The staffing, workload, and distribution of work between units has varied during this period, although the populations served have remained relatively constant.

It was standard practice in all the units to request necropsy when death was unexpected or when the diagnosis was in doubt. Often necropsy was not requested in patients in the "poor prognosis" categories listed below or when it was believed that the diagnosis had been clarified by recent operation or intensive investigations.

In analysing the data an individual judgment, based on the clinical description and the pathologist's report, was made as to whether the patient's outlook would have been favourable had embolism not occurred. Patients were classed as poor prognosis if they fell into one or more of the following categories: (a) over the age of $80,(b)$ malignant disease with metastases, or $(c)$ end-stage chronic disease such as obliterative arterial disease, respiratory or cardiac failure, or both. The remainder were categorised as good prognosis.

To determine whether trends in embolism were influenced by changes in the relative severity of the operations, the latter were divided into "major" and "minor." Major operations included all intra-abdominal or thoracoabdominal procedures, nephrectomy, cystectomy, prostatectomy, mastectomy, repair of incisional hernia, vascular grafts, endarterectomies, and amputation.

\section{Results}

The overall statistics are presented in the table. During the five years there were 61038 operations of which $16664(27 \%)$ were classed as major. Operations increased from around 10000 a year in 1959 to over 14000 in 1979 . There were 1528 postoperative deaths and 804 necropsies $(53 \%)$. Examination of the clinical particulars of the 724 in whom no necropsy was performed showed that $543(75 \%)$ were in the poor prognosis category as defined above, leaving 181 cases with a good prognosis ( $11.8 \%$ of all deaths) in whom information was based on clinical diagnosis alone. Seventeen emboli were reported in 1979 as compared with between 30 and 38 in each of the previous years.

The importance of these figures is obscure unless certain other factors are taken into account. These include: the pattern of surgery -for instance, the proportion of major cases; the operative mortality rates; the necropsy rates; and the accuracy of clinical diagnosis in cases where no necropsy was performed. These factors will be examined in turn.

\section{MAJOR OPERATIONS}

While the number of major operations rose during the period (table) the rise was less pronounced than the rise in the total number of operations. Thus major operations expressed as a percentage of the total number of operations show a modest decline. Since pulmonary embolism is usually associated with major surgery it would seem appropriate to express the incidence of embolism as a percentage of 
Overall statistics for 1959-79 inclusive

\begin{tabular}{lrrrrrr}
\hline & 1959 & 1964 & 1969 & 1974 & 1979 & Total \\
\hline Total No of operations & 10159 & 9987 & 11593 & 15066 & 14233 & 61038 \\
Major operations & 3020 & 2877 & 3429 & 3779 & 3559 & 16664 \\
Deaths & 351 & 298 & 322 & 300 & 257 & 1528 \\
Necropsies & 204 & 158 & 170 & 168 & 104 & 804 \\
Pulmonary embolism & 36 & 38 & 30 & 37 & 17 & 158 \\
\hline
\end{tabular}

the total number of major operations rather than of the total number of operations.

\section{OPERATIVE MORTALITY}

The mortality expressed as a percentage of all operations fell from $3.5 \%$ in 1959 to $1.8 \%$ in 1979 . Expressed as a percentage of major operations it fell from $11.6 \%$ in 1959 to $7 \cdot 2 \%$ in 1979 . Pulmonary embolism in addition to showing a fall in absolute numbers also fell in relation to the number of necropsies. It was reported in only $6.6 \%$ of necropsies in 1979 as compared with a range of $9.3 \%-12.8 \%$ in the earlier years.

\section{NECROPSY RATES}

Clearly the necropsy rate has an important influence on the reported incidence of embolism. The overall rate fell from $58 \%$ in 1959 to $40 \%$ in 1979 (fig 1). This fall was due to fewer necropsies being performed

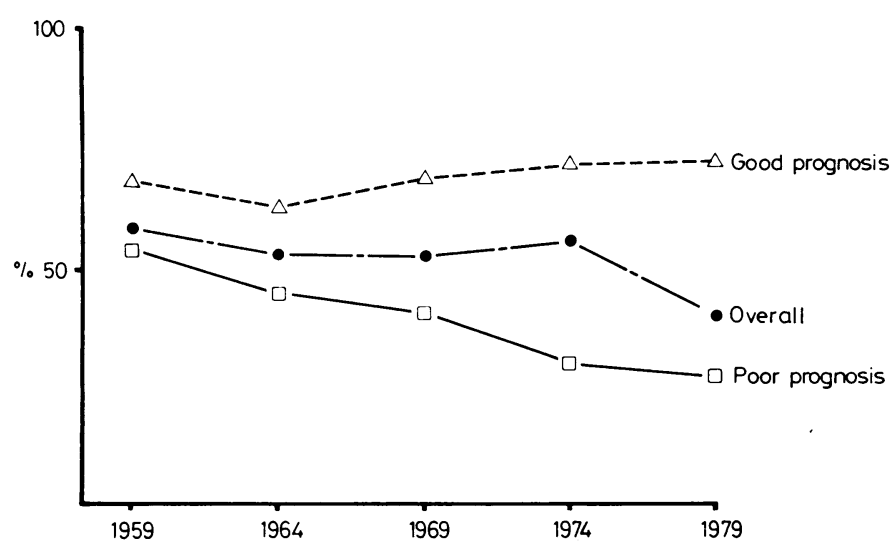

FIG 1 -Trends in necropsy rates.

in patients with a poor prognosis, for the necropsy rate in patients with a good prognosis actually rose slightly from $68 \%$ in 1959 to $72 \%$ in 1979. Thus comparisons confined to the group with a good prognosis-and this is the important group in terms of prevention-appear to be meaningful assuming that necropsy techniques remained relatively standard throughout the period. Discussion with pathologists has shown no change in routine necropsy techniques or methods of reporting.

\section{CLINICAL DIAGNOSIS AND NECROPSY FINDINGS}

The overall incidence of embolism reported here represents a combination of clinical and necropsy diagnosis. The unreliability of clinical diagnosis is well recognised. Since diagnostic accuracy would not be regarded as important for the later stages of care for patients dying of incurable disease, we shall consider only the group with a good prognosis. Pulmonary embolism was diagnosed before death in 92 patients. Of these, 70 underwent necropsy. Embolism was confirmed in $54(77 \%$ ) but not found in 15, giving a false-positive rate of $23 \%$. Out of the remaining 381 necropsies in this group-that is, patients with a good prognosis in whom a clinical diagnosis of embolism had not been made-pulmonary embolism as a cause of death was reported in 15-a false-negative rate of $4 \%$, and in two it was an incidental finding. Further analysis is therefore confined to the necropsy-proved group.

What were the trends when their various corrections were made? Figure 2 shows the trends in necropsy-proved embolism in patients with a good prognosis, expressed as per 100 major operations. There was a fall from $0.5 \%$ to $0.15 \%$ per 100 . When we looked at the types of patients dying of pulmonary embolism a change was apparent.

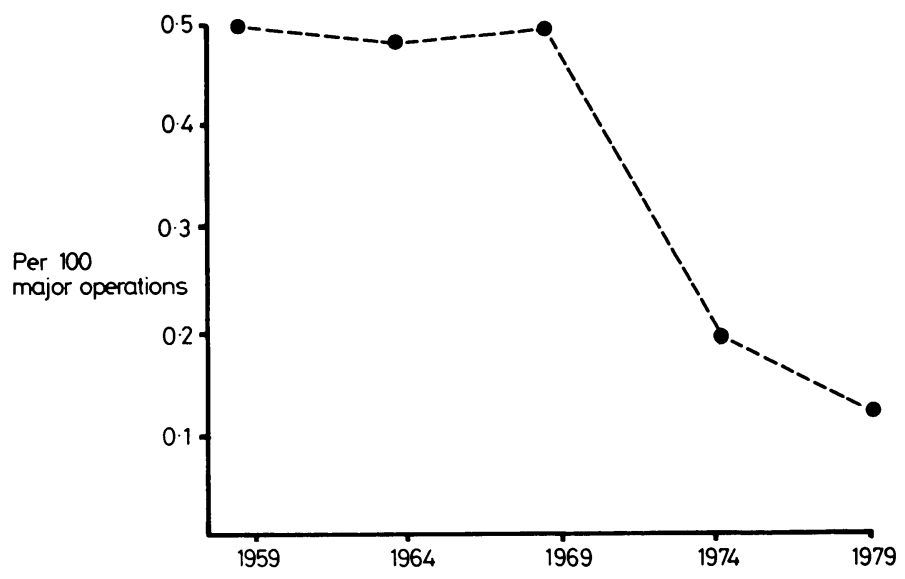

FIG 2-Necropsy-proved pulmonary embolism expressed as per 100 major operations in patients with a good prognosis.

Among the patients with a good prognosis who died in 1959, we found two after partial gastrectomy for peptic ulcer, five after cholecystectomy, two after simple division of adhesions, and one each after appendicectomy, prostatectomy, amputation, simple mastectomy, varicose vein ligations, left hemicolectomy, and dilatation of urethra. In 1979 there were only six deaths in the group with a good prognosis and these followed closure of perforated duodenal ulcer, subtotal gastrectomy for carcinoma, emergency aortic Y-graft, removal of infected aortic graft, and transurethral prostectomy-in general a group of far more serious cases. In 1979 there were no deaths from embolism after elective operations for non-malignant conditions. This difference was observed despite the rise in the number of operations, including major operations, noted earlier.

\section{Discussion}

The standardised method of setting out their reports adopted by the surgeons over the years has in part offset some of the difficulties and disadvantages of retrospective analysis.

It is well known that the frequency with which pulmonary embolism is reported is proportional to the diligence with which it is sought. In most patients it is an occult disease since most emboli are small and do not cause infarction. As for postmortem examination there is no doubt that painstaking search along the ramifications of the pulmonary arterial tree and serial sections through the lung will find emboli much in excess of the numbers reported here. ${ }^{5}$ These limitations on our data are fully accepted.

We were, however, concerned particularly with pulmonary embolism as a cause of death in surgical patients who ought, on other grounds, to have survived. Such emboli would be likely to be suspected clinically and to be found by routine necropsy methods. Furthermore, we were concerned with trends shown by methods at necropsy that have not been observed to change over this period.

Possibly the early detection of deep vein thrombosis or of herald emboli has resulted in therapeutic intervention and the prevention of deaths. This, however, does not accord with our clinical experience, which suggests that deep vein thrombosis is less common in surgical wards than formerly, nor with our understanding of the natural history of thromboembolism. We showed in a previous survey in the North Lothian District that in only $12 \%$ of patients who died of embolism did deep vein 
thrombosis give rise to clinically recognisable symptoms in the legs beforehand. ${ }^{6}$

We therefore believe that we have shown a real trend. In the first 15 years of the study (1959-74) the percentage of emboli per necropsy did not fall. Therefore the fall must have reflected the improving operative mortality rates. In 1979, however, in addition to the continued fall in operative mortality there was a fall in the proportion of necropsies at which embolism was reported and overall in the proportion of deaths in which embolism was reported by clinical or postmortem examination, or both.

There are many possible explanations for these trends. Throughout this period clinical turnover has accelerated, patients have been mobilised earlier, and discharged earlier. Indeed, we cannot exclude the possibility that some patients may have had fatal embolism after discharge home. Anaesthetic techniques have improved and in particular there has been greater use of local and regional anaesthesia.

Survey in the Edinburgh area in 1978 showed that three out of four general surgeons used routine deep vein thrombosis prophylaxis, of which the commonest was low dose subcutaneous heparin with or without elastic or mechanical compression. Such prophylactic methods were introduced in the early 1970s. It cannot be stated from this study whether prophylaxis has contributed to the fall (as we would like to believe) or whether we surgeons are all busily trying to prevent a disappearing disease.

We should like to acknowledge the help of Dr Rosamund Gruer, community medicine specialist, and $\mathrm{Mr} \mathrm{D}$ Gordon, research assistant, South Lothian District, in collecting data from the Edinburgh surgical audit. The information on which this study was based was compiled by many Edinburgh surgeons over a span of 20 years. We owe them all our thanks.

\section{References}

1 Soloff LA, Rodman T. Acute pulmonary embolism. Am Heart $\mathcal{f} 1967$;74: 710-21.

2 Dupont PA. The problem of deep venous thrombosis and pulmonary embolism. In: Nicolaides AN, ed. Thromboembolism. Lancaster: MTP Ltd, 1975:1-7.

${ }^{3}$ Ruckley CV. Venous thromboembolic disease. $f$ R Coll Surg Edinb 1975; 20:10-24.

4 Wolfe WG, Sabiston DC. Pulmonary embolism. In: Ebert PA, ed. Major problems in clinical surgery. Vol XXV. London: W B Saunders, 1980:9-16.

${ }^{5}$ Morrell T, Dunnell MS. The post-mortem incidence of pulmonary embolism in a hospital population. Br f Surg 1968;55:347-52.

${ }^{6}$ Macintyre IMC, Ruckley CV. A clinical and autopsy study of pulmonary embolism. Scott Med f 1974;19:20-4.

(Accepted 5 March 1982)
Is there any bronchial or pulmonary parenchymal hazard to people exposed to many years of inhalation of hot finely cut flakes of glass, sulphur fumes, starch spray, or coconut flour used in sheet glass manufacture?

All dusts and most gases are potentially harmful. Some are far more dangerous than others, the acute or chronic toxicity being influenced by biological half life, inhaled concentration, and duration of exposure. Also individual sensitivity will vary, especially in the case of those agents causing immunological reactions. Starch comes in the list of "nuisance particulates" but these are not inert, some cellular response being provoked in the lung if the inhaled concentration is sufficiently great. For this reason, nuisance dusts have a time-weighted eight-hour average inhalation threshold limit value of $10 \mathrm{mg} / \mathrm{m}^{31}$ at which concentration collagen is not formed to any appreciable extent and the tissue reaction is potentially reversible. Possibly, however, if a commercial grade is used the starch may still contain some proteinous matter that could cause immunological reactions. Coconut flour will contain higher amounts of protein. Flour and grain dusts are listed occupational agents for the causation of occupational asthma as a prescribed disease. ${ }^{2}$ It would not be unexpected for the starch and coconut flour to produce immunological effects in a small percentage of people exposed.

"Hot finely cut flakes of glass" are unlikely to be harmful if the exposures have been below the value recommended $\left(10 \mathrm{mg} / \mathrm{m}^{3}\right){ }^{1}$ The heat content is of no consequence. Particles above $20 \mu \mathrm{m}$ aerodynamic diameter will not enter the respiratory system, and by the time particles below this diameter are inhaled they will be cold because of the very high ratio of surface area to volume. Most reports on the health effects of glass refer to glass fibres, and finely cut flakes are unlikely to be more irritant than fibres. The Health and Safety Commission have proposed a control standard for glass fibre of $5 \mathrm{mg} / \mathrm{m}^{3}$ and 5 fibres $/ \mathrm{ml},{ }^{3}$ the latter figure being for fibres of a size able to enter the lung proper. The change in gravimetric standard proposed from $10 \mathrm{mg} / \mathrm{m}^{3}$ is primarily because of surface additives on the fibres. The fibre control standard does not apply to particulate glass. Therefore these proposals do not apply to the questioner's circumstances. Sulphur fumes will be gaseous and will, presumably, consist mainly of $\mathrm{SO}_{2}$. Because $\mathrm{SO}_{2}$ forms sulphurous acid in the respiratory system, irritation obviously occurs. Bronchoconstriction has been reported at a concentration as low as $1.6 \mathrm{ppm}$, and constant exposure to $1 \mathrm{ppm}$ has produced a reported accelerated loss of pulmonary function. ${ }^{3}$ Despite this the threshold limit value has been set at $2 \mathrm{ppm}$. The National Institute for Occupational Safety and Health in the United States has proposed a threshold limit value of
0.5 ppm, but this has not yet been accepted. ${ }^{4}$ At higher levels of exposure, chronic bronchitis and pulmonary oedema have been reported. It is good practice to keep all contaminants to as low a level as is reasonably practicable, the recommended hygiene standard being regarded as the upper desirable limit. The questioner should perhaps consider the advisability of obtaining an occupational hygiene survey to determine the extent of exposure and to consider whether it could be further lowered, as well as continuing clinical observation of the work force.-F W LUNAU, consultant in occupational hygiene, Kingston-upon-Thames.

${ }^{1}$ Health and Safety Executive. Threshold limit values. London: HMSO, 1979. (Guidance note EH15.)

Industrial Injuries Advisory Council. Occupational asthma. London: HMSO, 1981. Health and Safety Commission. Man-made mineral fibres. Report to Advisory Committee on Toxic Substances. London: HMSO, 1979.

American Conference of Governmental Industrial Hygienists. Documentation of threshold limit values. Cincinnati: ACGIH, 1980.

US Department of Health, Education and Welfare. Criteria for a recommended standard: occupational exposure to fibrous glass. Washington: DHEW, 1977. (DHEW (NIOSH) publication No 77-152.)

\section{What oral antibiotic is least likely to cause intestinal symptoms?}

Gastrointestinal symptoms often accompany oral administration of antibacterial drugs and can occur with all of these drugs. Whether or not they occur probably depends as much on the patient as on the particular drug used; and patients who develop symptoms with one antibiotic are more likely to develop them with others. Nevertheless, some differences in relative frequency have been described and may be used as a rough guide to choosing alternative drugs for patients who develop gastrointestinal symptoms. Diarrhoea is probably due in most patients to qualitative or quantitative changes in bowel flora, though direct toxic effects on the bowel mucosa and immunological factors may play a part $^{1}$; and it is more common with antibiotics that are incompletely absorbed. Thus the incidence is higher with penicillin $\mathrm{V}$, ampicillin, and tetracycline than with the newer derivatives of ampicillin-for example, amoxycillin and talampicillin ${ }^{2}$ - cephalexin, co-trimoxazole, and trimethoprim. Anorexia, nausea, and gastric irritation are less common with ampicillin and amoxycillin than with the ampicillin esters (talampicillin, pivampicillin), and may possibly be less common with oral cephalosporins and trimethoprim than with other antibacterials.-LINDA BEELEY, consultant pharmacologist, Birmingham.

1 Anonymous. Antibiotic diarrhoea. $\mathrm{Br}$ Med $\mathcal{F} 1975$;iv :243-4. Dukes MNG. Side effects of drugs annual 5. Amsterdam: Excerpta Medica, 1981. 261. 